Chirurg 2021 · 92:171

https://doi.org/10.1007/s00104-021-01357-1

Angenommen: 12. Januar 2021

Online publiziert: 2. Februar 2021

(c) Springer Medizin Verlag GmbH, ein Teil von Springer Nature 2021

\section{Originalpublikation.}

Quaas A, Schloesser H, Fuchs $H$, Zander T, Arolt C, Scheel AH, Rueschoff J, Bruns CJ, Buettner R, Schroeder W (2020) Improved tissue processing in esophageal adenocarcinoma after Ivor Lewis esophagectomy allows histological analysis in all surgically removed lymph nodes with significant effects on nodal UICC stages. Ann Surg Oncol (online publiziert)

Hintergrund und Fragestellung. Beim Ösophaguskarzinom sind die Anzahl der metastatisch befallenen sowie resezierten Lymphknoten (LK) und der daraus resultierende $\mathrm{pN}$-Status etablierte Faktoren der onkologischen Langzeitprognose. Die Zahl der histopathologisch diagnostizierten LK hängt hierbei von einer möglichen neoadjuvanten Vorbehandlung, dem Ausmaß der chirurgischen Lymphadenektomie, aber auch der pathologischen Aufarbeitung des chirurgischen Resektates ab. In der vorliegenden prospektiven Studie eines High-Volume-Zentrums wird eine erweiterte Aufarbeitung des Präparates analysiert, dies mit der Frage, ob sich die Anzahl der histologisch nachgewiesenen LK erhöht und ob sich damit das nodale UICC(Union for International Cancer Control)-Stadium verändert.

Methoden. Es wurden 77 konsekutive Patienten mit einem Adenokarzinom nach neoadjuvanter Therapie entsprechend dem CROSS- oder FLOT-Protokoll und einer standardisierten Ösophagektomie mit 2-Feld-Lymphadenektomie in die Untersuchung eingeschlossen. Nach Fixierung des Präparates in Formalin wurden alle palpablen LK (in der

W. Schröder · C. J. Bruns

Klinik für Allgemein-, Viszeral- und Tumorchirurgie, Universitätsklinik Köln, Köln, Deutschland

\title{
Pathologische Aufarbeitung des Resektates nach transthorakaler Ösophagektomie
}

Regel $>4 \mathrm{~mm}$ ) vom Resektat manuell separiert, in Paraffin eingebettet, nach H\&E(Hämatoxylin-Eosin)-Färbung mikroskopisch untersucht und nach UICC klassifiziert (Standardaufarbeitung). In der erweiterten Aufarbeitung wurde das verbliebene Fettgewebe vom Resektat vollständig entfernt und mittels Aceton-Kompressions-Technik weiterverarbeitet, um dann die identifizierten nodalen Strukturen nach Standard histopathologisch $\mathrm{zu}$ untersuchen und $\mathrm{zu}$ klassifizieren. Primäre Outcome-Variable war die Anzahl der detektierten LK nach standardisierter und erweiterter pathologischer Aufarbeitung.

Ergebnisse. Nach Standardaufarbeitung des Präparates wurden im Durchschnitt 23,1 LK histopathologisch identifiziert. Diese Anzahl stieg signifikant auf 40,5 LK an, wenn das verbliebene Fettgewebe mit der Aceton-Kompressions-Technik bearbeitet wurde $(p<0,0001)$. Der Anstieg war unabhängig vom Body-Mass-Index der untersuchten Patienten. Die Anzahl der metastatischen LK stieg ebenfalls signifikant von durchschnittlich 3,2 auf 4,2 LK mit der erweiterten Aufarbeitung an $(p<0,0001)$. Mit dem vermehrten Nachweis von positiven LK war bei $13 \%$ der Patienten eine Änderung des pNStatus zu höheren Stadien verbunden.

\section{Kommentar}

Die Untersuchung zeigt zum einen die allgemeine Bedeutung der histopathologischen Aufarbeitung des Präparates im Vergleich zur chirurgischen Lymphadenektomie. Zum anderen erlaubt die erweiterte Aufarbeitung mittels Aceton-
Kompressions-Technik eine zuverlässige Beurteilung der tatsächlich entfernten LK mit klinisch relevantem Einfluss auf das UICC-Stadium. Inwieweit sich hierdurch auch die onkologische Prognose der untersuchten Kollektive ändert, muss in weiteren Studien mit Analyse der Überlebensdaten geklärt werden.

\section{Korrespondenzadresse}

Prof. Dr. W. Schröder, FACS FEBS

Klinik für Allgemein-, Viszeral- und

Tumorchirurgie, Universitätsklinik Köln

Kerpener Str. 62, 50937 Köln, Deutschland

wolfgang.schroeder@uni-koeln.de

Interessenkonflikt. W.Schröder und C.J. Bruns geben an, dass kein Interessenkonflikt besteht. 